\title{
Participation in physical activity after consuming meals of differing caloric content does not alter appetite four hours post-activity
}

\begin{abstract}
The present study assessed children's appetite after consumption of either a lowcalorie (LC) or high-calorie (HC) meal, followed by a timed bout of physical activity. Children aged six to tenyears $(\mathrm{N}=19)$ participated in each of two trials, where they were asked to consume a $\mathrm{HC}$ or LC meal, and were then given free-choice access to physical and sedentary activities for 30 minutes. Trials were separated by one week, and treatment order of meals was randomized. The two, fast food-style meal conditions were comprised of the same food items and identical in macronutrient proportion, however were designed to contain approximately twice as much energy in the $\mathrm{HC}$ ( $\sim 600$ calories) compared to LC ( $\sim 300$ calories) condition. Subjective satiety ratings were collected via Visual Analogue Scale (VAS) at four time points during each trial (pre-meal, post-meal, post-activity, and four hours post-meal). ANOVA revealed a meal condition by time interaction $(\mathrm{p}=0.045)$ for Appetite rating. Compared to $\mathrm{HC}$, Appetite ratings were greater $(\mathrm{p} \leq 0.015)$ in LC both immediately post-meal $(71 \pm 74$, $\mathrm{HC}$ versus $131 \pm 96, \mathrm{LC})$ and post-activity session $(96 \pm 66 \mathrm{HC}, 160 \pm 57 \mathrm{LC})$. There were no differences $(\mathrm{p} \geq 0.16)$ in Appetite rating between the conditions at the pre-meal and four hours post-meal time points ( $p ' s \geq 0.16$ ). Therefore, while Appetite was greater immediately after eating the LC meal than the $\mathrm{HC}$ meal, that difference is eliminated by four hours post-meal which is likely to be the time the next meal is consumed.
\end{abstract}

Volume I Issue 4 - 2014

\author{
Samantha Uhas,' Rachael Pohle-Krauza, ${ }^{2}$ \\ Kelly Wagner,' Jacob E Barkley' \\ 'Exercise Science, Kent State University, USA \\ ${ }^{2}$ Department of Human Ecology, Youngstown State University, \\ USA
}

Correspondence: Jacob E Barkley, Exercise Science, Kent State University, I63E MACC Annex, 350 Midway Drive, Kent, OH 44242, USA, Tel 13306720209, Fax 13306722250, Email jbarkleI@kent.edu

Received:June 10,2014 | Published: July 19,2014

Keywords: exercise, appetite, physical activity, eating, children

\section{Abbreviations: HC, high calorie; LC, low calorie; VAS, visual analog scale}

\section{Introduction}

High-intensity exercise has been found to temporarily reduce appetite. ${ }^{1}$ While some previous evidence has indicated the effect of exercise on appetite is brief, several studies have demonstrated that appetite may be suppressed for an extended period of time by postmeal exercise in adults and adolescents. ${ }^{1,2}$ These previous studies have demonstrated that about of exercise can lead to a prolonged decrease in hunger, a delay before food is again desired and/or reduced caloric intake versus not exercising, up to three hours post-meal, relative to meal consumption alone. ${ }^{1-3}$ This greater satiation and reduced caloric intake that may occur after exercise may be attributed to potentiating of anorexigenic gut hormones such as glucagon like peptide-1 and pancreatic polypeptide. ${ }^{3,4}$

In addition to immediate caloric expenditure, the evidence supporting attenuation of appetite and/or reduced caloric intake postexercise could have important implications for preventing daily caloric surpluses. ${ }^{4}$ However, these effects have thus far only been assessed in adults and adolescents. Studying this effect in children is of importance, as $80 \%$ of overweight or obese children become overweight or obese adults. ${ }^{5}$ Therefore, better understanding of approaches that may reduce caloric intake in children is warranted and may have a significant, positive impact on the likelihood of the development of obesity in adulthood. If post-meal exercise reduces appetite in children it may be possible to maintain satiety after exercise even after a lower calorie meal. Such a finding could inform recommendations seeking to encourage healthy weight-control behaviors in children. In addition to daily caloric and exercise guidelines, new recommendations for healthy weight-control may also include the importance of postmeal exercise in appetite suppression, leading to lower daily caloric consumption. Presently, it is unknown if post-meal exercise can hold postprandial appetite constant across two meals of differing caloric content: one high calorie and one low calorie. If post-meal exercise aids in appetite control, it may be possible to feed exercising children lower calorie meals and maintain an appetite that is comparable to a higher calorie meal. This could have implications on total daily caloric consumption. Therefore, the purpose of the present study was to assess children's appetite for four hours after eating a low-calorie meal (LC) and participating in a bout of exercise, versus eating a highcalorie meal (HC) and participating in exercise. We hypothesized that children will respond in a similar manner to what has been previously demonstrated in adults and adolescents where post-meal exercise will have prolonged appetite-suppressing effects even after consuming a LC meal.

\section{Materials and methods}

Nineteen children ( $n=13$ male and six female), aged six to tenyears, reported to our laboratory twice in order to complete two experimental conditions (LC, HC). During the initial visit the parents/legal guardians and the children read and signed informed consent and assent forms, respectively. Children were free of any contraindication to physical activity (e.g. cardiovascular, metabolic, orthopedic disorders). All study procedures were approved by the Institutional Review Board at Kent State University. Laboratory visits were scheduled for the same day of the week, one week apart and for the same time, either 11:00 am or 12:00 pm Monday through Friday. Condition order (LC, HC) was randomly assigned. Participants were 
required to eat a similar breakfast no later than 9:00 am and participate in similar activity during mornings that preceded each trial. In order to confirm between-trial consistency parents were verbally asked whether or not their child had complied with these eating and activity specifications. If a child did not comply, they would have been sent home and rescheduled for the following week. There were no children that needed to be rescheduled. After obtaining consent and assent, children's height $(\mathrm{cm})$ and weight $(\mathrm{kg})$ were assessed with a balance beam scale (Health-O-Meter, Alsip, IL) and electronic stadiometer (Charder Electronic, Taipei, Taiwan), respectively. A member of research staff directed participants to complete a subjective assessment of Appetite (visual analogue scale, VAS) then to consume one of the two experimental meals. Children were instructed to consume meals in their entirety, or in case of refusal, to eat as much of the food as they could within the meal period (15minutes). Previous studies have indicated that watching television while eating may increase caloric consumption in children. ${ }^{6}$ To this end, participants were permitted to select age-appropriate DVDs (Scooby Doo ${ }^{\circledR}$ or Sponge Bob Square Pants $\left.{ }^{\circledR}\right)$ to view on a television located in the laboratory. At the end of the meal period, Appetite again was assessed via VAS.

After the immediately post-meal VAS assessment, children were taken to a 4,360 square foot gymnasium located within the same building as the laboratory where meals were consumed. In the gymnasium, children had free-choice access to physical and sedentary activities for 30minutes (described in section below) and total activity was monitored via a validated accelerometer (ACtigraph GT1M, Pensacola, Florida). ${ }^{7}$ Immediately following the activity period, children were asked to complete a third VAS Appetite assessment, then children and their parents were provided with a written reminder asking them to abstain from eating or drinking anything (except water) for a period of four hours after completion of the experimental meal. At the four-hour post-meal time point, children were instructed to complete the fourth and final appetite assessment VAS (provided to them by study staff in the earlier laboratory session). Upon completion of the final Appetite VAS children were free to eat as per normal family routine. Parents were instructed to ensure that their child did not eat until the final appetite assessment was completed and were asked to mail the final Appetite assessment VAS back to the laboratory in a postage-paid envelope provided. No parents indicated any difficulty in completing this final Appetite assessment as instructed and all final assessments were returned to the laboratory. The second trial was identical to the first except the participant consumed the opposite meal.

\section{Meal conditions}

Current dietary guidelines specify that daily energy requirements for children (aged 6-10) range from 1600 to $2200 \mathrm{kcal},{ }^{1}$ consistent with studies employing double-labeled water methods. Thus, our $\mathrm{HC}$ and LC test meals, (732 and 315kcal respectively) provided approximately $38 \%$ and $17 \%$ of estimated daily needs for participants (assuming 1900kcal diet). These energy provisions are either less than (LC condition) or equal to (HC condition) those provided in an average school lunch for children in this age group. ${ }^{8,9}$ Experimental meals contained $9 \%$ of daily protein, $47 \%$ of daily carbohydrates, and $44 \%$ of recommended daily fat intake. Meals consisted of Wendy's ${ }^{\circledR}$ chicken nuggets and French fries, Hawaiian ${ }^{\circledR}$ punch, and Heinz ${ }^{\circledR}$ ketchup. Food was weighed before and after each meal to obtain weight of all foods consumed. In the $\mathrm{HC}$ condition children were served 90 grams of chicken nuggets, 110 grams of French fries, 27 grams of ketchup, and 10 ounces of Hawaiian punch. In the LC condition children were served 36 grams of chicken nuggets, 44 grams of French fries, 27grams of ketchup, and 4 ounces of Hawaiian punch. Food for meals were purchased immediately prior to the session and reheated to 140 degrees before serving. The tray used to serve participants was organized the same way for each participant for each trial. The tray consisted of a bowl of chicken nuggets, a bowl of French fries, ketchup, a cup of Hawaiian punch, and napkins. Participants were able to request water if all Hawaiian punch was consumed. Participants had 15 minutes to consume the meal, and were given a stopwatch in order to aid with pacing of consumption. Research personnel provided verbal encouragement to children as needed, using phrases such as, "Can you eat one more chicken nugget?" or "Can you finish your juice?" If children were unable to finish, but consumed at least half of the presented meal they were allowed to continue with the study. While some children were not able to consume the $\mathrm{HC}$ meal in its entirety, children consumed an average of 55\% less energy in the LC (291.8 $\pm 12.1 \mathrm{kcals})$ compared to HC condition $(659.5 \pm 101.3 \mathrm{kcals})$.

\section{Gymnasium set-up}

Immediately following completion of the meal participants were fitted with the ACtigraph accelerometer and brought to the gymnasium for a 30minute free-choice play session. After the accelerometer was fitted to the child's waist using an elastic belt and instructions were given, research personnel interacted with the child only if they required assistance. Each child participated in the free-choice play condition independently. Other than a single member of the research team observing the child, there were no other individuals present in the gymnasium while each child was participating in the activity session. The gymnasium provided free-choice access to hurdles, jump rope, Nerf footballs, soccer balls, basketballs, and two obstacle courses made of gymnastics soft play equipment. A table and chair with sedentary alternatives was also available to the child. Sedentary alternatives included: blank paper and coloring utensils, genderappropriate dolls and figurines, structured games such as Jenga TM and Perfection TM, and crossword/word find puzzles. Children were instructed to sit in the provided chair if they wished to utilize any of the activities on the table. Children moved between physical activities and the sedentary table as much as they pleased for the 30minutes. Total per-minute accelerometer counts were recorded as the measure of physical activity behavior for each child. Accelerometer counts per child were then averaged across all participants for the high calorie and low calorie conditions separately.

\section{Measurement of appetite and subjective satiety}

Visual analog scale (VAS) booklets were utilized to assess measures of participants' satiety. The scale used is a 100-millimeter line with extreme opposite ratings of various components that influence satiety at either end of the line. Children were asked to mark a point on the line to which their sensation of each component corresponds. The marked points were then measured with a standard metric ruler to determine the VAS score for each satiety component at each time point. Criteria assessed included: prospective food consumption (or how much the child feels they could eat), hunger, fullness, as well as thirst and nausea (used as distracters). An appetite score was then calculated. The appetite score incorporated the three measured satiety scores (hunger, fullness and prospective food consumption) and was calculated using the following

Formula: Appetite Score=Hunger + Prospective Consumption $+(100$ - fullness) 
Participants completed a VAS appetite assessment booklet before each experimental meal, immediately after those meals, immediately after the 30minute activity sessions and four hours after the meals. This methodology for assessing appetite via VAS has been shown to be valid in adults and children. ${ }^{10,11}$ Research personnel read each criterion to the participant as to be sure the child understood the question and responded appropriately. The fourth booklet was sent home with the child and parents were coached on how and when to complete it and asked to bring it back for the second visit or provided with an envelope and stamp to mail it back to the lab. Satiety has similarly been evaluated using VAS in previous studies and these scales have been shown to be valid and reproducible, particularly in studies with repeated measurements such as this present study. ${ }^{12}$

\section{Analytical plan}

Participant's physical characteristics (age, height, weight) were compared between boys and girls using independent-samples $t$-tests. A paired-samples $t$-test was performed to compare accelerometer counts min-1 during the $\mathrm{HC}$ condition to the $\mathrm{LC}$ condition. A two sex (boys, girls) by two conditions (HC, LC) by four-time point (premeal, immediately post-meal, immediately post-activity, four hours post-meal) analysis of variance (ANOVA) with repeated measures on condition and time point was performed to assess changes in appetite scores. Post-hoc $t$-tests with the Benjamini and Hochberg ${ }^{13}$ False Discovery Rate correction for multiple comparisons were performed for any significant main or interaction effects.

\section{Results}

\section{Physical characteristics}

Physical characteristics are shown in Table 1. Boys $(8.6 \pm 1.1$ years old) were significantly $(p=0.004)$ older than girls $(6.7 \pm 1.1$ years old $)$. Boys $(136.3 \pm 7.7 \mathrm{~cm})$ were also significantly $(p=0.026)$ taller than girls $(125.2 \pm 12.0 \mathrm{~cm})$. There was no significant $(p \geq 0.16)$ difference in weight between boys $(32.6 \pm 9.6 \mathrm{~kg})$ and girls $(26.1 \pm 6.5 \mathrm{~kg})$. There was also no significant $(p \geq 0.54)$ difference in BMI between boys $\left(17.2 \pm 3.1 \mathrm{~kg} \cdot \mathrm{m}^{-2}\right)$ and girls $\left(16.4 \pm 1.0 \mathrm{~kg} \cdot \mathrm{m}^{-2}\right)$.

Table I Physical characteristics. Data are means \pm SD

\begin{tabular}{lll}
\hline & Boys & Girls \\
\hline Age $(\text { years })^{*}$ & $8.6 \pm 1.1$ & $6.7 \pm 1.1$ \\
Height $(\mathrm{cm})^{*}$ & $136.3 \pm 7.7$ & $125.2 \pm 12.0$ \\
Weight $(\mathrm{kg})$ & $32.6 \pm 9.6$ & $26.1 \pm 6.5$ \\
BMI $\left(\mathrm{kg} \cdot \mathrm{m}^{-2}\right)$ & $17.2 \pm 3.1$ & $16.4 \pm 1.0$ \\
\hline
\end{tabular}

*Significantly different between sexes

\section{Physical activity}

Physical activity during the HC $\left(2729 \pm 1066\right.$ counts $\left.\cdot \mathrm{min}^{-1}\right)$ and LC $\left(2457 \pm 1233\right.$ counts $\left.\cdot \mathrm{min}^{-1}\right)$ conditions was not different $(p=0.4)$.

\section{Appetite scores}

ANOVA revealed a significant $(p=0.045)$ meal condition (HC, LC) by time (pre, immediately post-meal, post-activity, four hours postmeal) interaction for appetite (Figure 1). Relative to the HC condition, appetite was greater $(p \leq 0.015$, power $=0.81)$ in the LC condition immediately post-meal $(71 \pm 74 \mathrm{HC}, 131 \pm 96 \mathrm{LC})$ and immediately post-activity session $(96 \pm 66 \mathrm{HC}, 160 \pm 57 \mathrm{LC}$, power 0.96). There were no differences $(p \geq 0.16)$ in appetite pre-meal $(225 \pm 63 \mathrm{HC}$, $222 \pm 61 \mathrm{LC}$, power $=0.06)$ or four hours post-meal $(234 \pm 69 \mathrm{HC}$, $259 \pm 52 \mathrm{LC}$, power $=0.26$ ) between the two meal conditions. There was an additional sex by time interaction $(p=0.02)$ as girls $(165 \pm 28)$ had greater $(p=0.02)$ appetite scores than boys $(109 \pm 49)$ at the postactivity time point and were not different $(p \geq 0.15)$ at any other time. There were no additional significant $(p \geq 0.08)$ main or interaction effects.

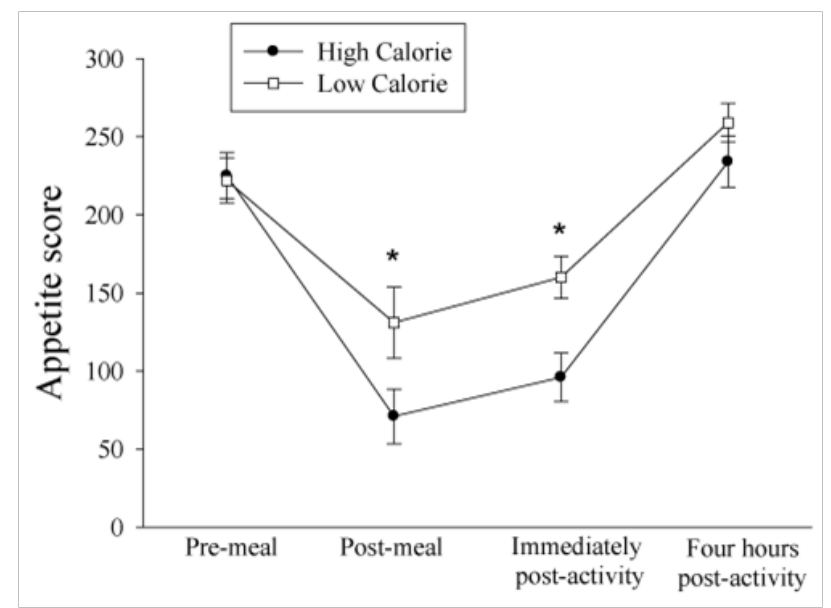

Figure I Mean $( \pm \mathrm{SE})$ appetite Scores for both low and high-calorie conditions across four-satiety questionnaire time points.

*Significant difference $(p<0.0 \mathrm{I})$ between conditions at these time points.

\section{Discussion}

While there is some evidence examining the effects of exercise on appetite and subsequent caloric consumption in adults and adolescents $^{1-3}$ we are not aware of any previous investigation that has examined the effect of exercise on controlling post-prandial appetite in children who consume meals of differing caloric contents. The present results indicated that children performing a bout of selfpaced exercise after eating a LC meal resulted in a four-hour postprandial appetite score that was similar to eating a $\mathrm{HC}$ meal followed by a similar bout of exercise. This would suggest that during the LC condition, by the time children would have been eating their next meal (i.e. dinner) their appetite was not different than it was in the $\mathrm{HC}$ condition.

This combined effect of exercise and eating on appetite regulation has previously been studied in adults and adolescents. Specifically, previous reports indicate that a high-intensity bout of exercise has suppressive effects on appetite in adults and caloric intake in adolescents. ${ }^{2,3}$ Possible mechanisms behind this effect may include changing satiety hormone levels. ${ }^{4}$ For example, glucagon like peptide-1, an appetite suppressive gut hormone, levels are greater during and following exercise than resting ${ }^{4}$ In addition, exercise has also been shown to elicit a significant increase in post-prandial plasma levels of pancreatic polypeptide in normal-weight men. ${ }^{4}$ Increases in glucagon like peptide- 1 and pancreatic polypeptide following exercise is a possible explanation for the prolonged suppressed appetite effects of exercise as both of these hormones reduce the sensation of hunger. Increased gut hormones and appetite regulating hormones may lead 
to increased satiety and decreased hunger. This may cause a delayed appetite for the next meal following acute exercise and less caloric consumption during the next meal, lowering daily caloric intake.

While this study shows that the four-hour appetite scores across the two conditions were similar, there are some limitations. The sample size is relatively small and does not allow for the comparison of overweight/obese and non-overweight children. Obesity-status may have an effect on the appetite scores in the present design. Overweight individuals have a lower concentration of glucagon like peptide-1 when compared to normal weight individuals. ${ }^{14}$ If glucagon like peptide-1 is reduced, appetite scores may be increased, as glucagon like peptide-1 is an appetite suppressant hormone. This may suggest that obese individuals need to exercise at a greater intensity or duration in order to achieve a similar amount of glucagon like peptide-1 secretion and satiety as lean individuals. The present study did not assess these hormones that are commonly linked to satiety, hunger and appetite (e.g. ghrelin, leptin, glucagon like peptide-1, and pancreatic polypeptide). Understanding the fluctuations in the hormones post meal and post exercise in children may provide a mechanism for the effects on appetite that were noted presently. Currently, the similar four-hour post-meal appetite scores between LC and HC suggest that caloric consumption at this point should be similar across the two conditions. However, the present study did not examine caloric intake, only appetite scores. Future research should measure caloric intake at the following meal (i.e. dinner). If the appetite score are predictive of actual caloric consumption, the ability of physical activity to blunt the appetite response in children may have significant benefits on controlling daily caloric intake. In addition, future studies may want to examine hormone levels, physical activity levels and caloric consumption if the caloric content of the meal was kept constant, but the percent of macronutrients (i.e. fat, protein, carbohydrate) were altered. For example, previous studies have demonstrated that pancreatic polypeptide increases during a high-protein meal and subsequently suppresses appetite,$^{15}$ if caloric content was kept the same, but the percent of protein present in the meal was greater, the participant may experience lower appetite than a lower-protein meal.

\section{Conclusion}

This was the first study we are aware of to assess the effect of a bout of exercise has on appetite after consuming meals of differing caloric content in children. Eating a LC meal followed by a bout of exercise resulted in an appetite that was very similar to a $\mathrm{HC}$ meal followed by a bout of exercise by the time the child would be eating their next meal (i.e. four-hour post experimental meal). In other words, children had a similar appetite at dinnertime regardless of their differing caloric intake at lunch. If the appetite scores are reflective of actual caloric consumption there would be no significant difference in caloric intake at the four-hour post meal time point between the $\mathrm{HC}$ and $\mathrm{LC}$ conditions. If children can reduce the number of calories consumed at lunch, continue expending a similar amount of calories and not have an increased appetite for their next meal, it increases the likelihood that they may avoid a positive daily energy balance which may, in turn, lead to weight loss and/or maintenance.

\section{Acknowledgments}

This project was supported through a SEED Grant from the College of Education Health and Human Services at Kent State University.

\section{Conflict of interest}

Author declares that there is no conflict of interest.

\section{References}

1. Sim AY, Wallman KE, Fairchild TJ, et al. High-intensity intermittent exercise attenuates ad-libitium energy intake. Int $J$ Obes (Lond). 2014;38(3):417-422.

2. Cheng MH, Bushnell D, Cannon DT, et al. Appetite regulation via exercise prior or subsequent to high-fat meal consumption. Appetite. 2009;52(1):193-198.

3. Thivel D, Isacco L, Montaurier C, et al. The 24-h energy intake of obese adolescents is spontaneously reduced after intensive exercise: a randomized controlled trial in calorimetric chambers. PLoS One. 2012;7(1):e29840.

4. Martins C, Morgan L, Truby H. A review of the effects of exercise on appetite regulation: an obesity perspective. Int $J$ Obes (Lond). 2008;32(9):1337-1347.

5. Burdette HL, Whitaker RC, Daniels SR. Parental report of outdoor playtime as a measure of physical activity in preschool-aged children. Arch Pediatr Adolesc Med. 2004;158(4):353-357.

6. Temple JL, Giacomelli AM, Kent KM, et al. Television watching increases motivated responding for food and energy intake in children. Am J Clin Nutr. 2007;85(2):355-361

7. Freedson P, Pober D, Janz KF. Calibration of accelerometer output for children. Med Sci Sports Exerc. 2005;37(11 Suppl):S523-S530.

8. Addison CC, Jenkins BW, White MS, et al. Examination of the food and nutrient content of school lunch menus of two school districts in Mississippi. Int J Environ Res Public Health. 2006;3(3):278-285.

9. Torun B. Energy requirements of children and adolescents. Public Health Nutr. 2005;8(7):968-993.

10. Mehra R, Tsalikian E, Chenard CA, et al. Feeding frequency and appetite in lean and obese prepubertal children. Obesity (Silver Spring). 2011;19(3):560-567.

11. Parker BA, Sturm K, MacIntosh CG, et al. Relation between food intake and visual analogue scale ratings of appetite and other sensations in healthy older and young subjects. Eur J Clin Nutr. 2004;58(2):212-218.

12. Flint A, Raben A, Blundell JE, et al. Reproducibility, power and validity of visual analogue scales in assessment of appetite sensations in single test meal studies. Int J Obes Relat Metab Disord. 2000;24(1):38-48.

13. Benjamini Y, Hochberg Y. Controlling the false discovery rate: a practical and powerful approach to multiple testing. JR Statist Soc B. 1995;57(1):289-300.

14. Chanoine JP, Mackelvie KJ, Barr SI, et al. GLP-1 and appetite responses to a meal in lean and overweight adolescents following exercise. Obesity (Silver Spring). 2008;16(1):202-204.

15. Lonovics J, Devitt P, Watson LC, et al. Pancreatic polypeptide: A review. Arch Surg. 1981;116(10):1256-1264. 